\title{
Estudio de la variabilidad genética en accesiones de papa (Solanum tuberosum L.) mediante marcadores SSRs
}

\section{Genetic variability study in accessions of papa (Solanum tuberosum L.) using markers SSRs}

Fecha de recepción: 21 de febrero de 2017

Manuel Alejandro Sánchez ${ }^{1}$

Fecha de aprobación: 21 de junio de 2017

DOI: http://doi.org/10.19053/01228420.v14.n2.2017.7150

\section{Resumen}

En este trabajo se determinó la variabilidad genética de 30 accesiones de papa nativas (Solanum tuberosum L.) del banco de germoplasma que administra la Corporación Colombiana de Investigación Agropecuaria (Corpoica), mediante cinco marcadores moleculares de tipo microsatélites (SSRs). En total, se identificaron 33 alelos con un rango entre 2 (STMS1049) hasta 10 (STMS1106), donde el contenido de información polimórfica (PIC) varió entre 0,368 (STM1049) hasta 0,574 (STM2022). El análisis de agrupamiento distribuyó las 30 accesiones de papas en siete grupos genéticos, con índices de similitud que oscilaron entre 0,452 (1231) hasta 0,841 (1439). A partir de los resultados obtenidos se puede concluir que con el uso de los marcadores moleculares empleados el material presentó variabilidad, poniendo en manifiesto el gran valor que tiene esta colección para los programas de mejoramiento genético de la especie.

Palabras clave: diversidad genética; germoplasma; marcadores moleculares; microsatélites; programas de mejoramiento genético.

\begin{abstract}
In the present paper, it was determined the genetic variability of 30 native potato accessions (Solanum tuberosum L.) from the germplasm bank, administered by the Colombian Agricultural Research Corporation (CORPOICA) by five molecular markers type microsatellites (SSRs). In total, were identified 33 alleles with a rank between 2 (STMS1049) to 10 (STMS1106), where the polymorphic information content (PIC) ranged from 0.368 (STM1049) to 0.574 (STM2022).
\end{abstract}

1 M.Sc. (c) Profesional independiente. manuel.27116218240@ucaldas.edu.co. 
The cluster analysis distributed the 30 potatoes accessions in seven genetic groups, with similarity indexes ranging from 0.452 (1231) to 0.841 (1439). From the results obtained, it can be concluded that with the use of the molecular markers employed, the material presented variability, showing the great value of this collection for the breeding programs improvement of the species.

Keywords: breeding programs improvement; genetic diversity; germplasm; microsatellites; molecular markers.

\section{Como citar:}

Sánchez MA. Estudio de la variabilidad genética en accesiones de papa (Solanum tuberosum L.) mediante marcadores SSRs. Rev. Cien. Agri. 2017; 14(2): 67-76. 


\section{Introducción}

La papa (Solanum tuberosum L.) pertenece a la familia de las solanáceas y al género Solanum (1); es una especie herbácea concentrada, principalmente, en la región Andina de Suramérica (2), donde se cultiva desde los 3.500 hasta los 4.200 m s.n.m., y constituye uno de los cultivos más importantes a nivel mundial, considerado alimento básico de aproximadamente 1.400 millones de personas (3). S. tuberosum ocupa el cuarto lugar en cuanto a producción, después del maíz, el arroz y el trigo (4), con una producción mundial, en 2014, de 385 millones de toneladas, producidas en 19 millones de hectáreas. Perú, Brasil y Argentina son los principales países latinoamericanos productores (4). En Colombia, la papa se cultiva entre los 2.000 y los 3.500 m s.n.m. (5) y anualmente se producen alrededor de 2.7 millones de toneladas, en un área de 144 mil hectáreas; Cundinamarca, Boyacá y Nariño son los principales departamentos productores (6).

Dada la variabilidad y adaptación de este cultivo, es necesario explorar la diversidad genética de las especies existentes, principalmente de aquellas mantenidas en bancos de germoplasma, donde se conservan y manejan genotipos que pueden tener alelos con características de interés agronómico, relacionados con producción y resistencia a estrés biótico y abiótico (7).

Los recursos genéticos de papa en Colombia son conservados y caracterizados por la Corporación Colombiana de Investigación Agropecuaria (Corpoica), que mantiene un total de 2.123 accesiones de S. tuberosum L. Este germoplasma se ha caracterizado a nivel genético empleando descriptores morfo-agronómicos; sin embargo, a nivel molecular es poca la información que se ha generado, y responde apenas al 1,88 \% del total de las accesiones de papa (8). No obstante, las caracterizaciones fenotípicas de los bancos de germoplasma deben ser complementadas con el uso de marcadores moleculares, que permiten evaluar la diversidad a nivel del ADN, el cual no es influenciado por el medioambiente (9).

Estudios sobre la diversidad genética en $S$. tuberosum L. han sido desarrollados para facilitar la utilización de los recursos genéticos usando técnicas moleculares. Por ejemplo, Tiwari et al. (10), usando 24 SSRs, caracterizaron 77 accesiones, encontrando una alta diversidad en la colección evaluada. Muhinyuza et al. (11) determinaron la relación genética entre genotipos de papa, usando 13 marcadores SSR en 18 muestras, obteniendo porcentajes de diversidad altos; del mismo modo, Ghebreslassie et al. (12) evaluaron una población de 63 muestras de S. tuberosum con 12 SSRs, identificando un alto nivel polimórfico entre los materiales estudiados.

En este contexto, y con el fin de facilitar un mejor aprovechamiento, desarrollar estrategias adecuadas de mejoramiento y conocer la diversidad de algunas accesiones de papa, este trabajo tuvo como objetivo estudiar la variabilidad genética basada en marcadores moleculares de tipo SSRs en 30 accesiones de $S$. tuberosum L., de la Colección Central Colombiana que administra Corpoica.

\section{Materiales y métodos}

\section{A. Material vegetal}

Se estudió una muestra total de 30 accesiones de papa pertenecientes a la Colección Central Colombia del banco de germoplasma de Corpoica-Tibaitatá (Tabla I). Los materiales se establecieron en un semillero de turba canadiense bajo condiciones de invernadero, con una temperatura promedio de 18 ${ }^{\circ} \mathrm{C}$. Este material se escogió dado que constituye el grupo de progenitores utilizados habitualmente en las actividades de mejoramiento. 
Tabla I. Accesiones de papa (Solanum tuberosum L.) del banco de Germoplasma- CORPOICA analizados en este estudio.

\begin{tabular}{|c|c|c|c|}
\hline Número & Código de colección & Ploidía & Procedencia \\
\hline 1 & 1951 & Tetraploide & Boyacá \\
\hline 2 & 1932 & Tetraploide & Boyacá \\
\hline 3 & 1286 & Tetraploide & Boyacá \\
\hline 4 & 1815 & Tetraploide & Boyacá \\
\hline 5 & 1295 & Tetraploide & Boyacá \\
\hline 6 & 2517 & Diploide & Boyacá \\
\hline 7 & 1641 & Tetraploide & Cundinamarca \\
\hline 8 & 1291 & Tetraploide & Cundinamarca \\
\hline 9 & 1273 & Tetraploide & Cundinamarca \\
\hline 10 & 1777 & Tetraploide & Cundinamarca \\
\hline 11 & 1336 & Tetraploide & Cundinamarca \\
\hline 12 & 1327 & Tetraploide & Cundinamarca \\
\hline 13 & 2039 & Tetraploide & Cundinamarca \\
\hline 14 & 2357 & Tetraploide & Cundinamarca \\
\hline 15 & 1469 & Tetraploide & Cundinamarca \\
\hline 16 & 2138 & Tetraploide & Cundinamarca \\
\hline 17 & 1439 & Tetraploide & Cundinamarca \\
\hline 18 & 1231 & Tetraploide & Cundinamarca \\
\hline 19 & 2071 & Tetraploide & Cundinamarca \\
\hline 20 & 1979 & Diploide & Cundinamarca \\
\hline 21 & 1445 & Tetraploide & Cundinamarca \\
\hline 22 & 1434 & Tetraploide & Cundinamarca \\
\hline 23 & 1632 & Tetraploide & Cundinamarca \\
\hline 24 & 2107 & Tetraploide & Nariño \\
\hline 25 & 1300 & Tetraploide & Nariño \\
\hline 26 & 0188 & Tetraploide & Nariño \\
\hline 27 & 1665 & Tetraploide & Nariño \\
\hline 28 & 1991 & Diploide & Nariño \\
\hline 29 & 2407 & Tetraploide & Nariño \\
\hline 30 & 1415 & Tetraploide & Nariño \\
\hline
\end{tabular}

\section{B. Extracción de $A D N$}

El ADN de cada una de las accesiones se obtuvo a partir de hojas jóvenes y tallos de plántulas germinadas en invernadero, utilizando el protocolo descrito por Doyle y Doyle (13). La calidad y concentración del ADN se verificó a través de geles de agarosa al $1 \%(\mathrm{p} / \mathrm{v})$ y espectrofotometría en relación con el grado de absorbancia (A260nm/ A280nm) en el equipo Nanodrop 2000 (Thermo Fisher Scientific, USA). El ADN se almacenó a -20 ${ }^{\circ} \mathrm{C}$ hasta su utilización. 


\section{Amplificación de SSRs}

La amplificacion de los cinco microsatélites (SSRs) por PCR se realizó con base en lo descrito por Ghislain (14). Las reacciones de PCR se llevaron a cabo en un volumen final de $20 \mu \mathrm{L}$, que incluyó: 50 ng de ADN; Buffer de PCR 1X (200mM de Tris$\mathrm{HCl}, \mathrm{pH} 8,4$ y $50 \mathrm{mM}$ de $\mathrm{KCl}$ ); $2,5 \mathrm{mM}$ de $\mathrm{MgCl}_{2}$; $0,5 \mu \mathrm{M}$ de cada marcador; $2 \mathrm{mM}$ de dNTPs, y $1 \mathrm{U}$ de Taq DNA polimerasa (Invitrogen). La reacción en cadena polimerada (PCR) se llevó a cabo en un termociclador PTC-100 TM MJ Research, empleando las siguientes condiciones de amplificación: un ciclo a $94{ }^{\circ} \mathrm{C}$, $3 \mathrm{~min} ; 30$ ciclos $\left[94{ }^{\circ} \mathrm{C}\right.$ por $60 \mathrm{~s}$, $72{ }^{\circ} \mathrm{C}$ por $\left.90 \mathrm{~s}\right]$; con una extensión de $72{ }^{\circ} \mathrm{C}$ por $8 \mathrm{~min}$. Los fragmentos amplificados se separaron a través de geles de poliacrilamida al $6 \%$ con 7 $\mathrm{M}$ urea corridos a $90 \mathrm{~V}$ por 45 min y teñidos con nitrato de plata (14).

\section{Análisis estadísticos}

Los fragmentos obtenidos por cada marcador se evaluaron visualmente. El tamaño de las bandas (alelos) se determinó por comparación de la movilidad en el gel con el marcador de peso $10 \mathrm{pb}$ (Invitrogen, Carlsbab, CA, USA). En cada marcador, un alelo correspondió a una banda amplificada; esta se registró como " 1 " o " 0 ", según su presencia o ausencia, respectivamente. La similitud genética entre los materiales se calculó utilizando el coeficiente de Nei y Li (15). En el programa NTSYS-PC, versión 2.2, se implementó un análisis de agrupamiento usando el método UPGMA (Mé- todo de agrupamiento de parejas por promedio no ponderado). En el programa GenAlex versión 6.5 Peakall y Somouse (16), la diversidad genética se estimó a través de los índices genéticos: Contenido de Información Polimórfica (PIC), Heterocigosidad Observada ( $\mathrm{Ho})$ y Heterocigosidad Insesgada $(\mathrm{Hi})$.

\section{Resultados y discusión}

\section{A. Extracción y cuantificación del ADN}

En las 30 accesiones se evaluó la eficiencia del protocolo de extracción de ADN, teniendo en cuenta la calidad y concentración del ADN obtenido. Para las muestras 12, 16 y 25 se evidenció un ADN medianamente degradado; esto podría atribuirse a la acción rápida de nucleasas, liberadas al iniciarse el proceso natural de degradación de los tejidos (17); un ejemplo de lo observado se muestra en la Figura 1. Por otro lado, en las muestras 20 y 24 se observan patrones de bandas poco nítidas, proceso posiblemente afectado por la baja estabilidad y la degradación a través del tiempo; sin embargo, esto no fue una limitante para la amplificación de las muestras, ya que las técnicas basadas en marcadores microsatélites requieren bajas cantidades de ADN (18).

En cuanto a la calidad, basada en el grado de absorbancia $(260 \mathrm{~nm} / 280 \mathrm{~nm})$, presentó valores de 2.00, lo cual indica una buena calidad del ADN extraído (19). Con respecto a la cantidad, se obtuvieron concentraciones con rango entre 481.9 (2517) y 6.942 (2138) ng/ $\mu \mathrm{l}$ (datos no mostrados).

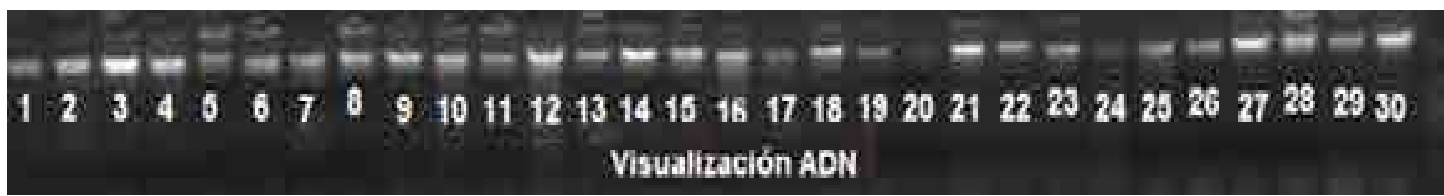

Fig 1. Visualización del ADN extraido de las accesiones en estudio, en gel de agarosa al $1 \%(\mathrm{p} / \mathrm{v})$ teñido con bromuro de etidio.

\section{B. Diversidad genética}

1) Análisis de la riqueza alélica: En total se encontraron 33 alelos, usando los 5 marcadores microsatélites analizados, con una variación desde 2 alelos por locus (STMS1049) hasta 10 (STMS1106) (Tabla
II); no obstante, estos valores no se acercaron a los reportadas por Hubert et al. (20) en la caracterización molecular de S. tuberosum, usando 10 marcadores de tipo SSR, en 11 variedades indias de papa, donde se identificaron un total 42 alelos. 
Liao y Guo (21), usando 24 SSRs en 85 cultivares, reportaron entre 5 y 19 alelos.

Del mismo modo, Onamu y Legaria (22) y Tierno y de Galarreta (23) reportaron en sus investigaciones entre 20 y 60 alelos, respectivamente; números altos si se comparan con esta investigación; estos valores se debieron, probablemente, a que usaron mayor número de marcadores y muestras. Por lo tanto, estas comparaciones sirven como punto de apoyo para corroborar la estimación de la variabilidad genética del material estudiado, teniendo en cuenta que los trabajos difieren en origen y evolución in situ, indicando que el número de alelos está directamente relacionado con la cantidad de muestras y el material de origen (24).
Se presentaron dos accesiones con alelos nulos por marcador, lo cual ocurrió con los marcadores STMS1052 y STMS0030. Por otra parte, las accesiones 1415 y 1777 presentaron alelos nulos con más de un marcador. En la accesión 1415 no hubo amplificación con los marcadores STMS2022 y STMS1049; mientras que para la accesión 1777 correspondió a los marcadores STMS1052 y STMS0030. Según Chambers y MacAvoy (25), inserciones, sustituciones o supresiones en el sitio de anclaje de los marcadores pueden impedir la amplificación del locus, produciendo un alelo nulo en una especie, debido a que la secuencia nucleotídica dentro de esta es diferente a la de la especie originaria.

Tabla II. Resumen de la diversidad genética de 30 accesiones de S. tuberosum L. PIC (Contenido de Información Polimórfica); Ho (Heterocigosidad Observada), Hi (Heterocigosidad Insesgada).

\begin{tabular}{clllll}
\hline Marcador & Cromosoma & PI C & Ho & Hi & Riqueza alélica \\
\hline STMS 2022 & II & 0,574 & 0,93 & 0,443 & 5 \\
STMS 1049 & I & 0,368 & 0,90 & 0,212 & 2 \\
STMS 1052 & VII & 0,568 & 0,83 & 0,242 & 7 \\
STMS 1106 & X & 0,557 & 0,92 & 0,331 & 10 \\
STMS 0030 & XII & 0,396 & 0,93 & 0,337 & 9 \\
\hline Todos los marcadores & - & $\mathbf{0 , 4 9 3}$ & $\mathbf{0 , 9 0 4}$ & $\mathbf{0 , 3 1 3}$ & $\mathbf{6 , 6}$ \\
\hline
\end{tabular}

\section{B. Contenido de Información Polimórfica (PIC)}

El Contenido de Información Polimórfica promedio para las accesiones evaluadas fue 0,493 , con rango de 0,368 a 0,574 , donde los marcadores STMS2022 $(0,574)$ y STMS1052 $(0,568)$ presentaron un mayor poder discriminatorio (Tabla II). El nivel promedio del PIC detectado por Cadima et al. (26) fue de 0,573 , en una colección comprendida en 1.476 accesiones de seis especies de papa cultivada; del mismo modo, De Galarreta et al. (27) obtuvieron un contenido polimórfico promedio de 0,521 en 19 variedades de papa de la Isla de la Palma, con 19 SSR, y Muthoni et al. (28) identificaron valores de PIC por encima de 0,65, usando 24 marcadores SRR en S. tuberosum; la diferencia de estos valores con los reportados en la presente investigación puede deberse a que sus estudios evaluaron mayor número de marcadores en una mayor población de diferente origen geográfico.

Sin embargo, el porcentaje de contenido polimórfico en este trabajo se asemeja a los reportados por Yujó et al. (29), quienes evaluaron la diversidad genética en Solanum tuberosum, Grupo Phureja, usando microsatélites SSRs, y obtuvieron un valor de PIC de 0,465; estos resultados indican que los marcadores evaluados para papa son informativos, logrando identificar niveles de variabilidad genética entre el material evaludado.

Otro índice reportado en la literatura para estimar la variabilidad genética es la heterocigosidad insesgada (HI); en este trabajo se encontró que esta heterocigosidad varió en un rango de 0,212 (STMS1049) a 0,443 (STMS2022), con una diversidad promedio de 0,313 (Tabla II). Navarro et al. 
(30), en el análisis de 19 poblaciones de Solanum tuberosum L. y Solanum phureja, con cinco marcadores de tipo RAMs, reportaron un valor promedio de 0,291 , lo que indica que existe una alta variabilidad genética dentro de la población; estos valores se aproximan a los obtenidos en esta investigación, al tratarse de condiciones similares relacionadas con el tamaño, la especie y la procedencia geográficamente de las poblaciones evaluadas.

Por otro lado, la heterocigosidad observada de menor y mayor valor se detectó para STMS 1056 $(0,83)$ y STMS $2022(0,93)$, respectivamente. Estos resultados son acordes con los reportados por Sharma y Nandineni (31), quienes, en el estudio de 47 variedades de papa de la India, utilizando un set de 23 cebadores de tipo SSR, obtuvieron valores entre 0,80 y 1,0 , indicando un alto número de heterocigotos, confirmando así que los resultados del presente estudio tienen gran valor como recurso fitogenético.
Esfahani et al. (32) mencionan que los valores de PIC no tienen relación con el número de bandas encontrado. En este trabajo esto fue evidente, ya que los marcadores con 9 y 10 alelos no presentaron valores PIC superiores a 0,574 . Por otro lado, los marcadores con valores PIC más bajos ( $\mathrm{PIC}<0,368)$ tuvieron relación con el bajo número de alelos detectados (igual a 2 alelos), de lo cual se podría inferir una baja capacidad discriminante de estos marcadores en la población analizada; sin embargo, entre los diferentes trabajos los valores del Índice de Contenido Polimórfico varían entre especies para un mismo marcador (14).

\section{Análisis de similitud genética}

El análisis de similaridad permitió observar las relaciones genéticas entre las diferentes accesiones evaluadas, que fueron distribuidas en siete grupos, con valores de similitud entre 0,452 y 0,841 (Figura 2).

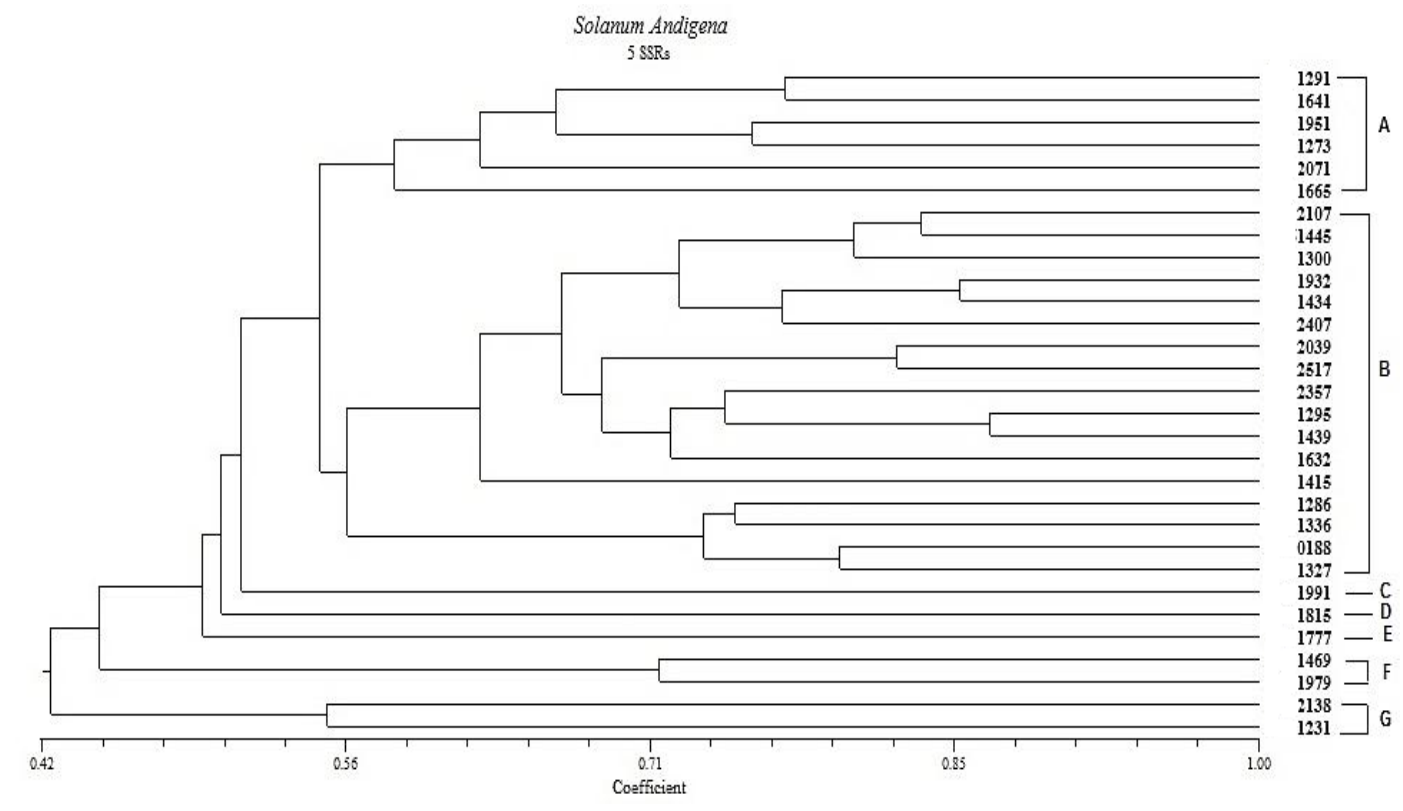

Fig 2. Dendrograma de 30 genotipos de papa S. tuberosum grupo andigemun, basado en similaridad genética utilizando el agrupamiento UPGMA.

En el grupo A se incluyen el $20 \%$ de las accesiones, con índices de similaridad superiores a $0,57, y$ en el grupo B, el $56.7 \%$, con distancias genéticas superiores a 0,56. A pesar de ser el grupo B el que más accesiones reúne de la misma zona, no las separó por ubicación geográfica, ya que se observa que accesiones con representantes de diferentes sitios de procedencia se localizan en el mimo grupo; además, tampoco separó los agrupamientos por ploidía tetraploide y diploide, ya que las accesio- 
nes de esta última se distribuyen entre los grupos de las primeras. Estos resultados concuerdan con lo reportado por Soto et al. (33). Este fenómeno se debe a que, posiblemente, la especie comparte muchos de estos marcadores genéticos, y quizá coinciden por sintenia o por ancestría (33), lo que sugiere ampliar el número de marcadores evaluados o el uso de técnicas moleculares que permitan detectar polimorfismos de un solo nucleótido (SNPs), y así se pueda identificar un mayor número de polimorfismo.

Tres muestras conformaron los grupos C (1991), D (1815) y $E$ (1777), que incluyen cada uno el 3.33 $\%$ de la población, con coeficientes de $0,53,0,51$ y 0,52 , respectivamente, lo que puede inferir que los marcadores usados permiten identificar una identidad genética única para estas muestras. Finalmente, las muestras restantes fueron incluidas en los grupos $F(1439 ; 1979)$ y $G(2138 ; 1231)$, que representan el 6,66 \%, con distancias entre 0,45 y 0,42 . Lo resultados aquí encontrados sugieren que los cinco pares de marcadores usados en este estudio son lo suficientemente informativos para identificar variaciones genéticas en algunas de las muestras analizadas.

Las distancias genéticas y los análisis de agrupamiento mostraron una similitud media de 0,65 entre las muestras analizadas. González y Peña (34), usando AFLPs en papas nativas, reportaron una similitud media de 0,75 ; este mayor nivel de similitud genética se puede atribuir a que González y Peña (34) incluyeron materiales de un solo departamento. Por otro lado, los resultados aquí indicados concuerdan con lo reportado por Soto (33), con nivel de similitud $=0,63$, lo que confirma la presencia de variabilidad genética entre el material evaluado.

El análisis genético y el número de bandas y porcentajes de polimorfismo observados evidenciaron el poder de los SSR en la diferenciación y asociación de accesiones estrechamente emparentadas. Estos marcadores se caracterizaron, además, por su nivel de polimorfismo y reproducibilidad. Del mismo modo, Bernal et al. (35); Oladosu et al. (36) y Cruz et al. (37) reportan en sus investigaciones resultados similares con cultivares diferentes. Por lo tanto, los datos obtenidos sugieren que existe un grado considerable de diversidad genética, y que cada material es útil para ser utilizado en programas de fitomejoramiento. Sin embargo, para futuros estudios de diversidad genética es recomendable incluir mayor número de accesiones y marcadores moleculares, con el fin de obtener una mejor precisión de la diversidad presente en los materiales del banco de germoplasma.

\section{Conclusiones}

El uso de los diferentes parámetros de diversidad permitió concluir que los materiales evaluados presentaron nivel de variabilidad genética. Así mismo, estos resultados ponen de manifiesto el gran valor que presentan estas accesiones; por lo tanto, es importante incorporar el análisis de marcadores SSR en los programas de premejoramiento genético de las colecciones de germoplasma.

\section{Agradecimientos}

A la Corporación Colombiana de Investigación Agropecuaria (Corpoica); al Ministerio de Agricultura, por su apoyo económico; a E. Sánchez, por su asistencia técnica; a J. Berdugo, por sus aportes críticos; a F. Rivera, por la lectura del documento, y a $V$. Núñez, por su dirección y enseñanza en la investigación.

\section{Referencias}

(1) Ovchinnikova A., Krylova E., Gavrilenko T., Smekalova T., Zhuk M., Knapp S., Spooner D. Taxonomy of cultivated potatoes (Solanum section Petota: Solanaceae). Rev Bot J Linn. Soc. 2011; 165(2): 107-155. DOI: http://doi.org/10.1111/j.10958339.2010.01107.x

(2) Bonierbale M., Amoros W., Espinoza J., Mihovilovich E., Roca W., Gómez R. Recursos genéticos de la papa: don del pasado, legado para el futuro. Rev ALAP. 2004; 1: 3-13.

(3) CIP, Centro Internacional de la Papa. 2016. Disponible en: http://cipotato.org/es.

(4) FAOSTAT. Food and Agriculture Organization of the United Nations. 2014. Disponible en: http://faostat.fao.org/site/339/default. aspx.

(5) Cucunubá LE. Diagnóstico del manejo ambiental del cultivo de la papa pastusa (solanum tuberosum), en un ecosistema de alta montaña, del municipio de Guatavita departamento de Cundinamarca. Tesis de Maestría, Facultad de Ciencias Contables Económicas y Administrativas, Universidad de Manizales, 2014. 
(6) AGRONET, Análisis-Estadísticas, Producción Nacional Por Producto. 2014. Disponible en: http://www.agronet.gov.co.

(7) Machida-Hirano R. Diversity of potato genetic resources. Rev Breed Sci. 2015; 65(1): 26-40. DOI: http://doi.org/10.1270/jsbbs.65.26.

(8) Valencia R., Lobo M., Ligarreto G. Estado del arte de los recursos genéticos vegetales en Colombia: Sistema de Bancos de Germoplasma. Rev Corpoica Cien Tecnol Agropecu. 2010; 11(1): 8594. DOI: http://doi.org/10.21930/rcta.vol11_num1_art:198.

(9) Osorio-Guarín JA. Caracterización molecular del banco de germoplasma de cacao (theobroma cacao, L.) del Instituto Amazónico de Investigaciones Científicas (SINCHI) mediante marcadores moleculares tipo microsatélites. Tesis de Grado, Facultad de Ciencias, Pontificia Universidad Javeriana, 2009.

(10) Tiwari J., Singh B., Gopal J., Poonam P., Patil V. Molecular characterization of the Indian Andigena potato core collection using microsatellite markers. Rev Afr J Biotechnol. 2013; 12:10. DOI: http://10.5897/AJB12.2436.

(11) Muhinyuza JB., Shimelis H., Melis R., Sibiya J., Gahakwa D., Nzaramba MN. Assessment of genetic relationship of promising potato genotypes grown in Rwanda using SSR markers. Rev Aust J Crop Sci. 2015; 9(8): 696.

(12) Ghebreslassie B., Githiri S., Mehari T., Kasili R., Ghislain M., Magembe E. Genetic diversity assessment of Farmers and Improved potato (Solanum tuberosum) Cultivars from Eritrea using Simple Sequence Repeat (SSR) markers. Rev Afr J Biotechnol. 2016; 15(35): 1883-1891. DOI: http://doi.org/10.5897/ AJB2016.15237.

(13) Doyle JJ., Doyle L. A rapid DNA isolation procedure for small quantities of fresh leaf tissue. Rev Phytochem Bull. 1987; 19: 11-15.

(14) Ghislain M., Andrade D., Rodriguez F., Hijmans R., Spooner D. Genetic analysis of the cultivated potato Solanum Tuberosum L. Phureja Group using RAPDs and nuclear SSRs. Rev Theor Appl Genet. 2006; 113: 1515-1527. DOI: http://doi.org/10.1007/ s00122-006-0399-7.

(15) Nei M., Li W. Mathematical Model for Studying Genetic Variation In Terms Of Restriction Endonucleases. Rev PNAS. 1978; 76: 5269-5273. DOI: http://doi.org/10.1073/pnas.76.10.5269.

(16) Peakall R., Smouse P. Genalex 6: Genetic Analysis in Excel. Population Genetic Software for Teaching and Research. Rev Mol Ecol. 2006; 6: 288-295. DOI: http://doi.org/10.1111/j.14718286.2005.01155.x.

(17) Hillis DM., Dixon MT. Ribosomal DNA: Molecular evolution and phylogenetic inference. Rev Q Rev Biol. 1991; 66: 411-453. DOI: http://doi.org/10.1086/417338.

(18) Muñoz-Flórez JE., Morillo-Coronado AC., Morillo-Coronado Y. Microsatélites amplificados al azar (RAM) en estudios de diversidad genética vegetal. Rev Acta Agron. 2008; 57(4): 219-226.

(19) San Juan A., Gálvez J., Plasencia M., Quirasco. Assessment of DNA extraction methods from various maize (Zea mays L.) tissues for environmental GMO monitoring in Mexico. Part I: Detectionbyend-point PCR. Rev Agrociencia. 2014; 48:17-33.

(20) Hubert GY., Gupta PH., Patel NJ., Shah AK., Acharya RR., Talati JG. Molecular characterization of Indian potato (Solanum tuberosum L.) Varieties for cold-induced sweetening using SSR markers. Rev J Plant Sci. 2015; 3(4): 191-196. DOI: http://doi. org/ 10.11648/j.jps.20150304.14.

(21) Liao H., Guo H. Using SSR to evaluate the genetic diversity of potato cultivars from Yunnan province (SWChina). Rev Acta Biol Craco Bota. 2014; 56: 16-27. DOI: http://doi.org/10.2478/abcsb-2014-0003.

(22) Onamu R., Legaria J. Diversidad genética entre variedades de papa (Solanum tuberosum L.) cultivadas en México usando marcadores RAPD e ISSR. Rev Mex Cien Agr. 2014; 5(4), 561-575.

(23) Tierno R., de Galarreta JIR. Characterization of high anthocyanin-producing tetraploid potato cultivars selected for breeding using morphological traits and microsatellite markers. Rev Plant Genet Resour. 2015; 1-10.

(24) Pacheco E., Arias D., Ojeda Z., Romero H. Diversidad y estructura genética de accesiones de palma de aceite (Elaeis guineesis Jacq.) provenientes de Camerún. Rev Colomb Biotecnol. 2014; 16(2): 57-67. DOI: http://doi.org/10.15446/rev.colomb. biote.v16n2.40132.

(25) Chambers GK., MacAvoy ES. Microsatellites: consensus and controversy. Comparative biochemistry and physiology Part B. Rev Biochem Mol Biol. 2000; 126(4): 455-476. DOI: http://doi. org/10.1016/S0305-0491(00)00233-9.

(26) Cadima X., Gabriel J., Veramendi S. Uso de marcadores moleculares microsatélite para el análisis de la diversidad genética de papa nativa de Bolivia. Rev J Sel Andi Res Soci. 2013; 4:1- 4.

(27) De Galarreta JR., Barandalla L., Lorenzo R., Gonzalez J., Rios DJ., Ritter E. Microsatellite variation in potato landraces from the island of La Palma. Revi Span J Agricu Res. 2013; 5(2), 186-192. DOI: http://doi.org/10.5424/sjar/2007052-5360.

(28) Muthoni J., Shimelis H., Melis R. Study of genetic relationship among Kenyan cultivated potato clones using SSR markers. Rev Aust J Crop Sci. 2014; 8(4), 502.

(29) Yujó D., Sarmiento F., Álvarez M., Brochero H., Gebhardt C., Mosquera T. Genetic diversity and population structure in diploid potatoes of group Phureja. Rev Crop Sci. 2015; 55(2): 760769. DOI: http://doi.org/10.2135/cropsci2014.07.0524.

(30) Navarro C., Bolaños C., Burbano T. Caracterización morfoagronómica y molecular de 19 genotipos de papa guata y chaucha (Solanum tuberosum L. y Solanum phureja Juz Et Buk) cultivados en el departamento de Nariño. Rev Cien Agr. 2010; 27(1): 27-39.

(31) Sharma V., Nandineni MR. Assessment of genetic diversity among Indian potato (Solanum tuberosum L.) collection using microsatellite and retrotransposon based marker systems. Rev Mol Phylogenet Evol. 2014; 73:10-17. DOI: http://doi.org/10.1016/j. ympev.2014.01.003.

(32) Esfahani ST., Shiran B., Balali G. AFLP markers for the assessment of genetic diversity in european and North American potato varieties cultivated in Iran. Rev Crop Breed Appli Biotechnol. 2009; 9: 75-86. DOI: http://doi.org/10.12702/1984-7033.v09n01a11.

(33) Soto J., Medina T., Aquino Y., Estrada R. Diversidad genética de papas nativas (Solanum spp.) conservadas en cultivares nativos del Perú. Rev Per Biol. 2013; 20(3): 215-222. 
(34) González J., Peña G. Caracterización molecular de papas nativas (Solanum spp.) del distrito de Chungui, Ayacucho, mediante AFLP. Rev Per Biol. 2014; 21: 277-282.

(35) Bernal-Parra N., Ocampo-Pérez J., Hernández-Fernández J. Caracterización y análisis de la variabilidad Genética de la granadiIla (passiflora ligularis juss.) en Colombia empleando marcadores microsatélites. Rev Bras Frutic. 2014; 36, 598-611. DOI: http:// doi.org/10.1590/0100-2945-251/13.

(36) Oladosu Y., Rafii M., Abdullah N., Malek M., Rahim H., Hussin G., Kareem I. Genetic variability and diversity of mutant rice revealed by quantitative traits and molecular markers. Rev Agrociencia. 2015; 49(3): 249-266

(37) Cruz E., Dantas AC., Carmo CD., Bastos LP. Molecular characterization of jaboticaba tree genotypes located in the municipalities of recôncavo of bahia. Rev Bras Frutic. 2016; 3: 38-39. DOI: http://doi.org/10.1590/0100-29452016510. 